\title{
Fractional Kramers Equation ${ }^{\dagger}$
}

\author{
E. Barkai* and R. J. Silbey \\ Department of Chemistry and Center for Materials Science and Engineering, Massachusetts Institute of \\ Technology, Cambridge, Massachusetts 02139
}

Received: September 29, 1999

\begin{abstract}
We introduce a fractional Kramers equation for a particle interacting with a thermal heat bath and external nonlinear force field. For the force-free case, the velocity damping follows the Mittag-Leffler relaxation and the diffusion is enhanced. The equation obeys the generalized Einstein relation, and its stationary solution is the Boltzmann distribution. Our results are compared to previous results on enhanced Lévy type of diffusion derived from stochastic collision models.
\end{abstract}

\section{Introduction}

The pioneering work of Scher and Montroll ${ }^{1}$ and Scher and $\mathrm{Lax}^{2}$ on the continuous time random walk ${ }^{3}$ applied to diffusion problems led to a revolution in our understanding of anomalous diffusion processes. Anomalous diffusion is now a wellestablished phenomenon, found in a broad range of fields. ${ }^{3-7}$ It is characterized by a mean-square displacement

$$
\left\langle x^{2}\right\rangle \sim t^{\delta}
$$

with $\delta \neq 1$. Various mechanisms are known to lead to enhanced diffusion $\delta>1$ or subdiffusion $\delta<1$. Usually such processes are non-Gaussian, meaning that the standard central limit theorem cannot be used to analyze the long-time behavior of these phenomena. To describe such anomalous processes, fractional kinetic equations were recently introduced by several authors. ${ }^{8-16}$ Within this approach, fractional space and/or time derivatives replace the ordinary time and/or space derivatives in the standard kinetic equation (e.g., Fokker-Planck equation). Examples include kinetics of viscoelastic media, ${ }^{9}$ Lévy flights in random environments, ${ }^{10}$ chaotic Hamiltonian dynamics, ${ }^{11}$ and quantum Lévy processes. ${ }^{16}$ For a discussion on Lévy statistics and continuous time random walk in the context of single molecule spectroscopy, see refs $17-19$.

In this paper, we introduce a fractional Kramers equation describing both the velocity $v$ and coordinate $x$ of a particle exhibiting anomalous diffusion in an external force field $F(x)$. In the absence of the external force field, the equation describes enhanced diffusion. The new equation we propose is

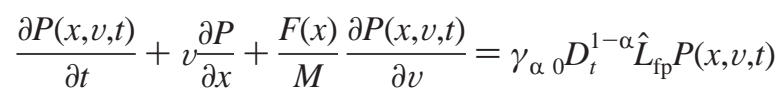

with $0<\alpha<1$ and

$$
\hat{L}_{\mathrm{fp}}=\frac{\partial}{\partial v} v+\frac{k_{\mathrm{b}} T}{M} \frac{\partial^{2}}{\partial v^{2}}
$$

the dimensionless Fokker-Planck operator. Here we employ the fractional Liouville-Riemann operator ${ }^{20}{ }_{0} D_{t}^{1-\alpha}$ in eq 1.2,

† Part of the special issue "Harvey Scher Festschrift".

* Corresponding author. Fax: +1-617-253-7030. E-mail: barkai@mit.edu. which we define later in eq 2.4. $\gamma_{\alpha}$ is a damping coefficient whose units are $\left[1 / \mathrm{s}^{\alpha}\right]$. In the presence of a bounding force $F(x)$ $=-V^{\prime}(x)$ the stationary solution of the fractional Kramers equation (1.2) is the Maxwell-Boltzmann distribution. When $\alpha=1$, we recover the standard Kramers equation.

Fractional kinetic equations are related to Montroll-Weiss continuous time random walk (CTRW). ${ }^{21-24}$ Here we show that the fractional Kramers equation is related to the coupled CTRW in the enhanced diffusion regime $\delta>1$. This case corresponds to the so-called Lévy walks that are observed in a number of systems. ${ }^{4,5}$ The different limits of the CTRW were used to model diverse physical phenomenon (when $F(x)=0$ ), and therefore fractional kinetic equations, in general, and the fractional Kramers equation, in particular, are believed to be of physical significance.

The basis of the fractional Kramers equation is the fractional Ornstein-Uhlenbeck process described by the fractional Fokker-Planck equation

$$
\frac{\partial Q(v, t)}{\partial t}=\gamma_{\alpha 0} D_{t}^{1-\alpha} \hat{L}_{\mathrm{fp}} Q(v, t)
$$

$Q(v, t)$ is the probability density of finding the particle at time $t$ with velocity $v$ when $F(x)=0$. We see that the fractional Kramers equation is a natural extension of eq 1.4 in which the streaming terms describing Newtonian evolution are added in the standard way (i.e., as in the Boltzmann or Liouville equations). When $\alpha=1$, we get the standard OrnsteinUhlenbeck process which has a fundamental role in nonequilibrium statistical mechanics.

The Rayleigh model was used to derive eq 1.4 for the normal case of $\alpha=1 .{ }^{25,26}$ The Rayleigh model for Brownian motion, also called the Rayleigh piston, considers a one-dimensional heavy particle of mass $M$ colliding with light noninteracting bath particles of mass $m$ which are always thermalized. According to this model, the moments of time intervals between collision events are finite. What happens when the variance of time intervals between collision events diverges? In this case we anticipate a non-Gaussian behavior. This case has been investigated by Barkai and Fleurov, ${ }^{27,28}$ and as we shall show in subsection II.B, for a nonstationary model, such an anomalous case corresponds to the fractional Fokker-Planck equation (1.4). However, as we shall show, the usual Rayleigh limit $m / M \rightarrow 0$ 
in the non-Gaussian case is not as straightforward as that for the ordinary Gaussian case.

The more fundamental question of the necessary conditions for transport to be described by diverging variance of time intervals between collision events, or how to derive $\alpha$ from first principles is not addressed in this paper. In this context we note that several mechanisms in which the variance of time between collisions (or turning events in random walk schemes) diverge and which lead to anomalous type of diffusion are known in the literature. ${ }^{4,5,7}$

Previous approaches ${ }^{8-15}$ have considered fractional kinetic equations in which the coordinate and/or time acquires the fractional character while in our approach the velocities are the variables that acquire fractional character. For $F(x)=0$ we find $1 \leq \delta=2-\alpha \leq 2$. The lower bound $\delta=1$ corresponds to normal diffusion, and the upper bound $\delta=2$ can also be easily understood. For a system close to thermal equilibrium we expect

$$
\left\langle x^{2}\right\rangle \leq\left(k_{\mathrm{b}} T / M\right) t^{2}
$$

and hence $\delta \leq 2$.

Previously Kusnezov et al. ${ }^{16}$ have suggested a fractional Kramers equation which was derived for the classical limit of a quantum Lévy process. Also in this work the velocities do acquire fractional character; however, this approach is very different from ours, because it is based on Reisz fractional operators and gives $\left\langle x^{2}(t)\right\rangle=\infty$ (i.e., $\delta=\infty$ ). Recently Metzler and Klafter ${ }^{29}$ considered a fractional kinetic equation that they also called a fractional Kramers equation. Their equation describes subdiffusion $(\delta<1)$ and is very different from our equation which described enhanced diffusion $(\delta>1)$.

This paper is organized as follows. In section II we introduce the fractional Ornstein-Uhlenbeck process described by eq 1.4, a brief introduction to fractional calculus is given. In subsection II.A the solution of the fractional Fokker-Planck equation (1.4) is presented, and in subsection II.B we derive this equation based on stochastic collision model. In section III we consider the fractional Kramers equation (1.2), general properties of this equation are given, and the force-free case is investigated in some detail. We end the paper with a short summary in section IV.

\section{Fractional Ornstein-Uhlenbeck Process}

Let $Q(v, t)$ be the probability density describing the velocity $v$ of a macroscopic Brownian particle, with mass $M$, interacting with a thermal heat bath. The Fokker-Planck equation describing the time evolution of $Q(v, t)$ with initial conditions $Q(v, t=0)$ is given by 26,30

$$
\frac{\partial Q(v, t)}{\partial t}=\gamma_{1} \hat{L}_{\mathrm{fp}} Q(v, t)
$$

We shall call such a Fokker-Planck equation standard or ordinary. According to eq 2.1, the damping law for the averaged velocity is linear, $\langle\dot{v}(\mathrm{t})\rangle=-\gamma_{1}\langle v(t)\rangle$, and the velocity fluctuations are thermal. The stationary solution of eq 2.1 is Maxwell's density defined with the thermal energy $k_{\mathrm{b}} T$. The corresponding Langevin equation is

$$
\dot{v}=-\gamma_{1} v+\xi(t)
$$

and $\xi(t)$ is Gaussian white noise. ${ }^{26,30}$ The stochastic process described by the Langevin equation is the well-known Ornstein-Uhlenbeck process. Originally eq 2.1 was derived by Rayleigh $^{25}$ for a particle interacting with a gas consisting of light particles; however, the scope of eq 2.1 is much wider. It is used to model Brownian motion in dense environments when memory effects are negligible.

In this section we generalize the Fokker-Planck equation (2.1) using fractional calculus. First, we give some mathematical definitions and tools.

The Liouville-Riemann fractional integral operator ${ }^{20}$ of order $\alpha>0$ is defined by

$$
{ }_{0} D_{t}^{-\alpha} f(t) \equiv \int_{0}^{t} \frac{\left(t-t^{\prime}\right)^{\alpha-1}}{\Gamma(\alpha)} f\left(t^{\prime}\right) \mathrm{d} t^{\prime}
$$

For integer values $\alpha=n ;{ }_{0} D_{t}^{-n}$ is the Riemann integral operator of order $n$. Fractional differentiation of order $\alpha>0$ is defined by

$$
{ }_{0} D_{t}^{\alpha} f(t) \equiv \frac{\mathrm{d}^{n}}{\mathrm{~d} t^{n}}\left[{ }_{0} D_{t}^{\alpha-n} f(t)\right]
$$

where $n-1 \leq \alpha<n$ and $\mathrm{d}^{n} / \mathrm{d} t^{n}$ is ordinary differentiation of order $n$. Within this fractional calculus

$$
{ }_{0} D_{t}^{ \pm \alpha} t^{\mu}=\frac{\Gamma(\mu+1)}{\Gamma(\mu \mp \alpha+1)} t^{\mu \mp \alpha}
$$

when $\mu>-1$. Notice that ${ }_{0} D_{t}^{ \pm \alpha} 1 \sim t^{\mp \alpha}$ when $0<\alpha<1$. The Laplace transform

$$
f(u)=\int_{0}^{\infty} \mathrm{e}^{-u t} f(t) \mathrm{d} t=\mathrm{L}[f(t)]
$$

of a fractional Liouville-Riemann operator is

$$
\mathrm{L}\left[{ }_{0} D_{t}^{\alpha} f(t)\right]=u^{\alpha} f(u)-\left.\sum_{k=0}^{n-1} u_{0}^{k} D_{t}^{\alpha-1-k} f(t)\right|_{t=0}
$$

$n$ is an integer satisfying $n-1<\alpha \leq n$. For fractional integrals $\alpha \leq 0$ the sum on the right-hand side of eq 2.7 vanishes. From eq 2.7 we see that the Laplace transform of a fractional derivative of $f(t)$ depends on fractional derivatives of that function at time $t=0$. In this work we use the convention that the arguments of a function indicate the space in which the function is defined; e.g., the Laplace transform of $Q(v, t)$ is $Q(v, u)$. form

The Fokker-Planck equation (2.1) is rewritten in the integral

$$
Q(v, t)-Q(v, t=0)=\gamma_{10} D_{t}^{-1} \hat{L}_{\mathrm{fp}} Q(v, t)
$$

We now replace the integer integral operator ${ }_{0} D_{t}^{-1}$ in eq 2.8 with a fractional integral operator ${ }_{0} D_{t}^{-\alpha}$ and $0<\alpha \leq 1$. The result is

$$
Q(v, t)-Q(v, t=0)=\gamma_{\alpha 0} D_{t}^{-\alpha} \hat{L}_{\mathrm{fp}} Q(v, t)
$$

where $\gamma_{\alpha}$ is a generalized damping coefficient with units $[\mathrm{s}]^{-\alpha}$. Ordinary differentiation of eq 2.9 gives the fractional FokkerPlanck equation

$$
\frac{\partial Q(v, t)}{\partial t}=\gamma_{\alpha 0} D_{t}^{1-\alpha}\left[\frac{\partial}{\partial v} v+\frac{k_{\mathrm{b}} T \partial^{2}}{M \partial v^{2}}\right] Q(v, t)
$$


When $\alpha=1$, the ordinary Fokker-Planck equation (2.1) is obtained. In eq 2.10 we use the natural boundary conditions

$$
\lim _{v \rightarrow \pm \infty} Q(v, t)=\lim _{v \rightarrow \pm \infty} \partial Q(v, t) / \partial v=0
$$

Later we shall show that the solution of eq 2.10 is non-negative and normalized. Equation 2.10 describes a fractional OrnsteinUhlenbeck process. When the velocity $v$ is replaced with a coordinate $x$, the equation describes an anomalously overdamped harmonic oscillator, as investigated in ref 13.

Equtions 2.9 and 2.10 are initial value problems. While eq 2.9 depends on a single initial condition $[Q(v, t=0)$ on the lefthand side of the equation], in solving eq 2.10 two initial conditions have to be specified, ${ }^{20}$ these being $Q(v, t=0)$ and 0 $\left.D_{t}^{-\alpha} Q(v, t)\right|_{t=0}$. When setting $\left.{ }_{0} D_{t}^{-\alpha} Q(v, t)\right|_{t=0}$ equal to zero, the two equations are equivalent. ${ }^{31}$

Multiplying eq 2.10 by $v$ and integrating over $v$, we find that the mean velocity is described by

$$
\langle\dot{v}(t)\rangle=-\gamma_{\alpha 0} D_{t}^{1-\alpha}\langle v(t)\rangle
$$

In Laplace space equation 2.12 reads

$$
\langle v(u)\rangle=\frac{v_{0}}{u+\gamma_{\alpha} u^{1-\alpha}}
$$

$v_{0}$ is the initial velocity. The inverse Laplace transform of eq 2.13 is

$$
\langle v(t)\rangle=v_{0} E_{\alpha}\left(-\gamma_{\alpha} t^{\alpha}\right)
$$

and

$$
E_{\alpha}(z)=\sum_{n=0}^{\infty} \frac{z^{n}}{\Gamma(1+\alpha n)}
$$

is the Mittag-Leffler function. When $\alpha=1$, the Mittag-Leffler reduces to the exponential. For large $t$, eq 2.14 exhibits a power law decay

$$
\langle v(t)\rangle \sim \frac{v_{0}\left(\gamma_{\alpha} t\right)^{-\alpha}}{\Gamma(1-\alpha)}
$$

and for short times the relaxation is a stretched exponential

$$
\langle v(t)\rangle \simeq v_{0} \exp \left[-\frac{\gamma_{\alpha} t^{\alpha}}{\Gamma(1+\alpha)}\right]
$$

In a similar way we find the second moment

$$
\left\langle v^{2}(t)\right\rangle=v_{0}^{2} E_{\alpha}\left(-2 \gamma_{\alpha} t^{\alpha}\right)+\frac{k_{\mathrm{b}} T}{M}\left[1-E_{\alpha}\left(-2 \gamma_{\alpha} t^{\alpha}\right)\right]
$$

exhibiting power law decay toward the thermal equilibrium value $\left\langle v^{2}(t=\infty)\right\rangle=k_{\mathrm{b}} T / M$. From eqs 2.14 and 2.18 , we see that the Mittag-Leffler relaxation replaces the ordinary exponential relaxation found for ordinary Brownian motion. These equations were derived in ref 27 based upon a stochastic collision model which we will discuss in subsection II.B.

A. Solution. Our aims are (a) to find a solution of the fractional Fokker-Planck equation (2.10) and (b) show that $Q(v, t)$ in eq 2.10 is a probability density. The solution we find is an integral of a product of two well-known functions. We use the initial conditions $Q(v, t=0)=\delta\left(v-v_{0}\right)$; the generalization for other initial conditions is carried out in the usual way.
We first show that the solution is normalized. The Laplace transform of eq 2.10 is

$$
u Q(v, u)-\delta\left(v-v_{0}\right)=\gamma_{\alpha} u^{1-\alpha} \hat{L}_{\mathrm{fp}} Q(v, u)
$$

Integrating eq 2.19 with respect to $v$, using the boundary conditions in eq 2.11 and the normalized initial condition, we find

$$
\int_{-\infty}^{\infty} Q(v, u) \mathrm{d} v=\frac{1}{u}
$$

Because $\mathrm{L}(1, u)=1 / u$, we see that $Q(v, t)$ in eq 2.10 is normalized.

Let us now find the solution in Laplace $u$ space. We write $Q(v, u)$ as

$$
Q(v, u)=\int_{0}^{\infty} R_{s}(u) G_{s}(v) \mathrm{d} s
$$

where

$$
\hat{L}_{\mathrm{fp}} G_{s}(v)=\frac{\partial}{\partial s} G_{s}(v)
$$

and

$$
G_{0}(v)=\delta\left(v-v_{0}\right)
$$

Equation 2.22 is the dimensionless ordinary Fokker-Planck equation (2.1), with solution ${ }^{26,30}$

$$
G_{s}(v)=\frac{\sqrt{M}}{\sqrt{2 \pi k_{\mathrm{b}} T\left(1-\mathrm{e}^{-2 s}\right)}} \exp \left[-\frac{M\left(v-v_{0} \mathrm{e}^{-s}\right)^{2}}{2 k_{\mathrm{b}} T\left(1-\mathrm{e}^{-2 s}\right)}\right]
$$

We see that $G_{s}(v)$ is a non-negative probability density function, describing the standard Ornstein-Uhlenbeck process and normalized according to

$$
\int_{-\infty}^{\infty} G_{s}(v) \mathrm{d} v=1
$$

$R_{s}(u)$ in eq 2.21 must satisfy a normalization condition. Using eqs 2.20 and 2.25 , we have

$$
\int_{0}^{\infty} R_{s}(u) \mathrm{d} s=\frac{1}{u}
$$

Inserting eq 2.21 in eq 2.19 , using eq 2.22 , and integrating by parts, we find

$$
\begin{gathered}
u \int_{0}^{\infty} R_{s}(u) G_{s}(v) \mathrm{d} s-\delta\left(v-v_{0}\right)= \\
\gamma_{\alpha} u^{1-\alpha} \int_{0}^{\infty} R_{s}(u) \hat{L}_{\mathrm{fp}} G_{s}(v) \mathrm{d} s=\gamma_{\alpha} u^{1-\alpha} \int_{0}^{\infty} R_{s}(u) \frac{\partial}{\partial s} G_{s}(v) \mathrm{d} s= \\
\gamma_{\alpha} u^{1-\alpha}\left[R_{\infty}(u) G_{\infty}(v)-R_{0}(u) G_{0}(v)\right]- \\
\gamma_{\alpha} u^{1-\alpha} \int_{0}^{\infty}\left[\frac{\partial}{\partial s} R_{s}(u)\right] G_{s}(v) \mathrm{d} s
\end{gathered}
$$

According to eq 2.26, the boundary term $R_{\infty}(u)$ in eq 2.27 is zero. Using the initial condition eq 2.23 in eq 2.27, we find

$$
\begin{aligned}
& \int_{0}^{\infty}\left\{u R_{s}(u)+\gamma_{\alpha} u^{1-\alpha}\left[\frac{\partial}{\partial s} R_{s}(u)\right]\right\} G_{s}(v) \mathrm{d} s= \\
& {\left[1-\gamma_{\alpha} u^{1-\alpha} R_{0}(s)\right] \delta\left(v-v_{0}\right)}
\end{aligned}
$$


Equation 2.28 is solved once both sides of it are equal to zero; therefore, two conditions must be satisfied, the first being

$$
\gamma_{\alpha} u^{1-\alpha} R_{0}(u)=1
$$

and the second being

$$
-\gamma_{\alpha} u^{1-\alpha} \frac{\partial}{\partial s} R_{s}(u)=u R_{s}(u)
$$

The solution of eq 2.30 with the condition eq 2.29 is

$$
R_{s}(u)=\frac{1}{\gamma_{\alpha} u^{1-\alpha}} \exp \left(-\frac{s u^{\alpha}}{\gamma_{\alpha}}\right)
$$

It is easy to check that $R_{s}(u)$ is normalized according to eq 2.6.

The solution of the problem in $t$ space is the inverse Laplace of eq 2.21

$$
Q(v, t)=\int_{0}^{\infty} R_{s}(t) G_{s}(v) \mathrm{d} s
$$

where $R_{S}(t)$ is the inverse Laplace transform of $R_{s}(u)$ given by

$$
R_{s}(t)=\frac{1}{\alpha \gamma_{\alpha} t^{\alpha}} z^{\alpha+1} l_{\alpha}(z)
$$

and

$$
z=\frac{\left(\gamma_{\alpha}\right)^{1 / \alpha} t}{s^{1 / \alpha}}
$$

Properties of $R_{s}(t)$ are discussed by Saichev and Zaslavsky. ${ }^{12}$ $l_{\alpha}(z)$ in eq 2.33 is a one-sided Lévy stable density, ${ }^{32}$ whose Laplace transform is

$$
l_{\alpha}(u)=\int_{0}^{\infty} \exp (-u z) l_{\alpha}(z) \mathrm{d} z=\exp \left(-u^{\alpha}\right)
$$

The proof that $R_{S}(t)$ eq 2.33 and $R_{s}(u)$ eq 2.31 are a Laplace pair is given in Appendix A (and see also ref 12).

A few features of the solution eq 2.32 can now be discussed. An interpretation of eq 2.32 in terms of a stochastic collision model will be given in the next subsection.

When $0<\alpha \leq 1, R_{s}(t)$ is a probability density normalized according to $\int_{0}^{\infty} \mathrm{d} s R_{s}(t)=1$. Because $G_{s}(v)$ is also a probability density, the solution eq 2.32 is normalized and nonnegative. This justifies our interpretation of $Q(v, t)$ as a probability density.

When $\alpha=1$, the solution reduces to the well-known solution of the ordinary Fokker-Planck equation. To see this, note that the inverse Laplace of eq 2.31 for $\alpha=1$ is $R_{s}(t)=1 / \gamma_{1} \delta(t-$ $\left.s / \gamma_{1}\right)$ and then use definition eq 2.32. When $\alpha=1 / 2$, we have

$$
l_{1 / 2}(z)=\frac{1}{2 \sqrt{\pi}} z^{-3 / 2} \exp \left(-\frac{1}{4 z}\right)
$$

with $z>0$, and then $R_{s}(t)$ eq 2.33 is a one-sided Gaussian

$$
R_{s}(t)=\sqrt{\frac{1}{\pi \gamma_{1 / 2}{ }^{2} t}} \exp \left(-\frac{s^{2}}{4 \gamma_{1 / 2}^{2} t}\right)
$$

Two other closed forms of one-sided Lévy probability densities $l_{2 / 3}(z)$ and $l_{1 / 3}(z)$ can be found in refs 33 and 34 . Series expansions of the Lévy stable density are in Feller's book, ${ }^{32}$ Chapter XVII.6.

In Figure 1, we show the solution for the case $\alpha=1 / 2$ and for different times. The solution is found with numerical integration of eq 2.32 using eqs 2.24 and 2.37. We choose the initial condition $Q(v, t=0)=\delta(v-1)$ (i.e., $\left.v_{0}=1\right)$ and $k_{\mathrm{b}} T / M$ $=1$. The solution exhibits a slow power law decay toward thermal equilibrium. We observe a cusp at $v=v_{0}=1$; thus, initial conditions have a strong signature on the shape of $Q(v, t)$. A close look at the figure shows that for short times (i.e., $t \leq$ 2 ) the peak of $Q(v, t)$ is at $v=v_{0}$. This is very different from Gaussian evolution for which the peak is always on $\langle v(t)\rangle$.

Metzler et al. ${ }^{13}$ have shown that a fractional Fokker-Planck equation, which describes subdiffusion $\delta<1$, can be solved using an eigenfunction expansion which is identical with the ordinary expansion of the Fokker-Planck solution; ${ }^{30}$ but in which the exponential relaxation of eigenmodes is replaced with a Mittag-Leffler relaxation. We can use the eigenfunction expansion in ref 13 to find a second representation of $Q(v, t)$ in terms of a sum of Hermite polynomials. Expanding $G_{s}(v)$, using the standard eigenfunction technique of Fokker-Planck solutions, we write

$$
\begin{aligned}
& G_{s}(v)= \sqrt{\frac{M}{2 \pi k_{\mathrm{b}} T}} \exp \left(-\frac{M v^{2}}{2 k_{\mathrm{b}} T}\right) \sum_{n=0}^{\infty} \frac{1}{2^{n} n !} \times \\
& H_{n}\left(\sqrt{\frac{M}{2 k_{\mathrm{b}} T}}\right) H_{n}\left(\sqrt{\frac{M}{2 k_{\mathrm{b}} T}} v_{0}\right) \exp (-n s)
\end{aligned}
$$

where $H_{n}$ are Hermite polynomials. We insert the expansion eq 2.38 in eq 2.32 and use

$$
\int_{0}^{\infty} R_{s}(t) \exp (-n s) \mathrm{d} s=E_{\alpha}\left(-n \gamma_{\alpha} t^{\alpha}\right)
$$

to find the eigenfunction expansion

$$
\begin{aligned}
& Q(v, t)= \sqrt{\frac{M}{2 \pi k_{\mathrm{b}} T}} \exp \left(-\frac{M v^{2}}{2 k_{\mathrm{b}} T}\right) \sum_{n=0}^{\infty} \frac{1}{2^{n} n !} \times \\
& H_{n}\left(\sqrt{\frac{M}{2 k_{\mathrm{b}} T}} v\right) H_{n}\left(\sqrt{\frac{M}{2 k_{\mathrm{b}} T}} v_{0}\right) E_{\alpha}\left(-n \gamma_{\alpha} t^{\alpha}\right)
\end{aligned}
$$

The stationary solution, determined by the smallest eigenvalue $n=0$, is the Maxwell distribution, which is independent of $\gamma_{\alpha}$ and $\alpha$.

An extension of the fractional Fokker-Planck equation 2.10 to higher dimensions is carried out by replacing the onedimensional Fokker-Planck operator (eq 1.2) with the appropriate $d$-dimensional Fokker-Planck operator (e.g., replace $\partial / \partial v$ with $\nabla)$. The solution for such a $d$-dimensional equation is then found to be eq 2.32 in which eq 2.24 must be replaced with the appropriate solution of the $d$-dimensional ordinary FokkerPlanck equation.

B. Collision Model. As mentioned above, the ordinary Fokker-Planck eq 2.1 was derived by Rayleigh over a century ago. Briefly, the Rayleigh model for Brownian motion considers a one-dimensional test particle with mass $M$ colliding with bath particles of mass $m$ and $\epsilon \equiv m / M \ll 1$. When collisions are frequent but weak, the ordinary Fokker-Planck equation is valid. ${ }^{26}$ Here we consider the case when the concept of collision 


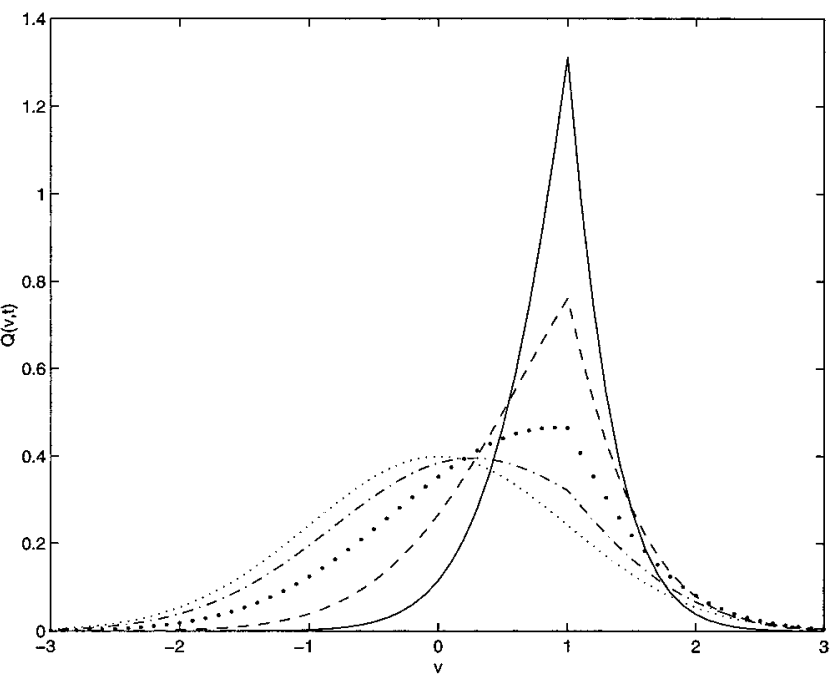

Figure 1. Dynamics of $Q(v, t)$ for the fractional Ornstein-Uhlenbeck process with $\alpha=1 / 2$ and for times $t=0.02,0.2,2$, and 20 (solid, dashed, dotted, and dot-dashed lines, respectively). Also shown (fine dotted curve) is the stationary solution, which is Maxwell's distribution. Notice the cusp on $v=v_{0}=1$ as well as the nonsymmetrical shape of $Q(v, t)$.

rate does not hold and the mean of the time intervals between collision events diverges. As mentioned in the Introduction, this case was investigated in refs 27 and 28 and as we shall show now such a case corresponds to the fractional Fokker-Planck equation 2.10.

We consider a particle of mass $M$ which moves freely in one dimension and at random times it collides elastically with bath particles of mass $m$. Bath particles are assumed to be much faster than the test particle. Collisions are elastic and one-dimensional and therefore the velocity $V_{M}^{+}$of the test particle immediately after a collision event can be related to the velocity $V_{M}^{-}$of the test particle just before the collision event according to

$$
V_{M}^{+}=\left(\frac{1-\epsilon}{1+\epsilon}\right) V_{M}^{-}+\frac{2 \epsilon}{1+\epsilon} \tilde{v}_{m}
$$

where $\epsilon=m / M$ and $\tilde{v}_{m}$ is the velocity of bath particle distributed according to Maxwell's distribution.

The times between collision events are assumed to be independent identically distributed random variables, implying that the number of collisions in a time interval $(0, t)$ is a renewal process. This is reasonable when the bath particles thermalize very quickly and when the test particle is slow. According to these assumptions, the times between collision events $\left\{\tau_{i}\right\}$ are described by a probability density $\psi(\tau)$, which is independent of the mechanical state of the test particle. Therefore, the process is characterized by free motion with constant velocity for time $\tau_{1}$, then a collision event described by eq 2.41 , then a free evolution for a period $\tau_{2}$, then again a collision, etc. The most important ingredient of the model is the assumption that $\psi(\tau)$ decays such as a power law for long time

$$
\psi(\tau) \sim \tau^{-1-\alpha}
$$

with $0<\alpha<1$. From eq 2.42 we learn that the mean time between collisions diverges, $\int_{0}^{\infty} \tau \psi(\tau) \mathrm{d} \tau=\infty$. Because in such a problem there is no characteristic time scale, the number of collisions in an interval $(0, t)$ is not proportional to $t$ for large times. In other words, the law of large numbers is not valid for the choice eq 2.42, leading to non-normal behavior. Similar waiting time distributions were used within the CTRW to model anomalous diffusion for the past 3 decades. When $\psi(\tau)$ is exponential and in the presence of an external force field $F(x)$, this model was investigated extensively in the context of reaction rate theory. ${ }^{35-41}$

Such a model is nonstationary, and the probability of a collision event in a small time interval $(t, t+\mathrm{d} t)$ is time dependent even in the limit of large times. This is a consequence of the diverging first moment.

Let $Q_{\text {col }}(v, t)$ be the probability density for finding the test particle with velocity $v$ at time $t$ and initially $v=v_{0}$. Using the model assumptions

$$
Q_{\mathrm{col}}(v, t)=\sum_{s=0}^{\infty} \tilde{R}_{s}(t) \tilde{G}_{s}(v)
$$

with $\tilde{R}_{s}(t)$ the probability that $s$ collision events have occurred in the interval $(0, t)$ and $\widetilde{G}_{s}(v)$ the conditional probability density of finding the particle with velocity $v$ after $s$ collision events. We note that eq 2.43 is the discrete version of eq 2.32 .

Using the map eq 2.41, it can be shown that

$\tilde{G}_{s}(v)=\frac{\sqrt{M}}{\sqrt{2 \pi k_{\mathrm{b}} T\left(1-\mu_{1}^{2 s}\right)}} \exp \left[-\frac{M\left(v-v_{0} \mu_{1}^{s}\right)^{2}}{2 k_{\mathrm{b}} T\left(1-\mu_{1}^{2 s}\right)}\right]$

with $\mu_{1}=(1-\epsilon) /(1+\epsilon)$. Not surprisingly $\tilde{G}_{s}(v)$ is Gaussian because velocities of colliding particles are Gaussian random variables. We note that

$$
\frac{\partial \tilde{G}_{s}(v)}{\partial s}=\ln \left(\mu_{1}^{-1}\right) \hat{L}_{\mathrm{fp}} \tilde{G}_{s}(v)
$$

with the initial condition

$$
\tilde{G}_{0}(v)=\delta\left(v-v_{0}\right)
$$

and $\ln \left(\mu_{1}^{-1}\right) \sim 2 \epsilon$. Equation 2.45 is an ordinary Fokker-Planck equation in which $s$, the collision number, plays the role of dimensionless time.

The Laplace $t \rightarrow u$ transform of $\tilde{R}_{s}(t), \tilde{R}_{s}(u)$, can be calculated using renewal theory

$$
\tilde{R}_{s}(u)=\frac{1-\psi(u)}{u} \psi^{s}(u)
$$

and $\psi(u)=\mathrm{L}[\psi(\tau)]$. From eq 2.43 we have in Laplace $t \rightarrow u$ space

$$
Q_{c o l}(v, u)=\sum_{s=0}^{\infty} \tilde{R}_{s}(u) \tilde{G}_{s}(v)
$$

Multiplying this equation from the left with $\hat{L}_{\mathrm{fp}}$, using eq 2.45 , and integrating by parts, we have

$$
\begin{array}{r}
\hat{L}_{\mathrm{fp}} Q_{\mathrm{col}}(v, u)=\frac{1}{2 \epsilon} \sum_{s=0}^{\infty} \tilde{R}_{s}(u) \frac{\partial \tilde{G}_{s}(v)}{\partial s}=-\frac{1}{2 \epsilon} \tilde{R}_{0}(u) \delta\left(v-v_{0}\right)- \\
\frac{1}{2 \epsilon_{s}=0} \sum^{\infty}\left[\frac{\partial}{\partial s} \tilde{R}_{s}(u)\right] \tilde{G}_{s}(v)
\end{array}
$$

We have used eq 2.46 and the boundary condition $\tilde{R}_{\infty}(u)=0$ for $\mathrm{u} \neq 0$ 
According to eq 2.47

$$
\frac{\partial}{\partial s} \tilde{R}_{s}(u)=\ln [\psi(u)] \tilde{R}_{s}(u)
$$

and because according to eq $2.42 \psi(\tau) \sim \tau^{-1-\alpha}$, we have

$$
\psi(u) \sim 1-A u^{\alpha}
$$

(here $A$ is a parameter with units $t^{\alpha}$ ) valid for small $u$, inserting eq 2.51 in eq 2.50 , we have

$$
\frac{\partial}{\partial s} \tilde{R}_{s}(u) \sim-A u^{\alpha} R_{s}(u)
$$

and from eq 2.47

$$
\tilde{R}_{0}(u) \sim A u^{\alpha-1}
$$

We are now ready to derive the fractional Fokker-Planck equation. Inserting eqs 2.52 and 2.53 in eq 2.49 , we find in the limit of small $A u^{\alpha}$ and $\epsilon$

$$
u Q_{\mathrm{col}}(v, u)-\delta\left(v-v_{0}\right)=\gamma_{\alpha} u^{1-\alpha} \hat{L}_{\mathrm{fp}} Q_{\mathrm{col}}(v, u)
$$

with

$$
\gamma_{\alpha}=\lim _{A \rightarrow 0, \epsilon \rightarrow 0} \frac{2 \epsilon}{A}
$$

Equation 2.54 is the fractional Fokker-Planck equation (2.1) in Laplace space. We note that the moments of the collision model in the limit $\epsilon \rightarrow 0$ found in ref 27 are identical with those found here based upon the fractional Fokker-Planck equations (2.14) and (2.18) as they should be.

\section{Fractional Kramers Equation}

Let $P(x, v, t)$ be the joint probability density function describing both the position $x$ and the velocity $v$ of a Brownian particle subjected to an external force field $F(x)$. The one-dimensional Kramers equation models such stochastic motion according to

$\frac{\partial P(x, v, t)}{\partial t}+v \frac{\partial P}{\partial x}+\frac{F(x)}{M} \frac{\partial P(x, v, t)}{\partial v}=\gamma_{1} \hat{L}_{\mathrm{fp}} P(x, v, t)$

where the Fokker-Planck operator $\hat{L}_{\mathrm{fp}}$ is given in eq 1.3. The Kramers equation (3.1) implies that noise is white and Gaussian, and it describes underdamped motion close to thermal equilibrium. Equation 3.1 is an extension of eq 2.1 which includes the coordinate $x$ as well as the effects of $F(x)$. We generalize Kramers equation in the same way as above and consider

$$
\begin{gathered}
\frac{\partial P(x, v, t)}{\partial t}+v \frac{\partial P(x, v, t)}{\partial x}+\frac{F(x)}{M} \frac{\partial P(x, v, t)}{\partial v}= \\
\gamma_{\alpha 0} D_{t}^{1-\alpha} \hat{L}_{\mathrm{fp}} P(x, v, t)
\end{gathered}
$$

with $0<\alpha<1$. The terms on the left-hand side of the equation are the standard streaming terms describing reversible dynamics according to Newton's second law of motion. The term on the right-hand side of the equation describes an interaction with a bath; it can be considered as a generalized collision operator replacing the ordinary collision operator found in the standard Fokker-Planck equation. As mentioned in the Introduction, the stationary solution of eq 3.2 is the Maxwell-Boltzmann distribution, and when $\alpha=1$, we recover the ordinary Kramers equation.
A formal solution of the fractional Kramers equation 3.2 can be found in terms of the solution of the ordinary Kramers equation. We denote the solution of the fractional Kramers equation with $P_{\alpha}\left(x, v, t, \gamma_{\alpha}\right)$ instead of $P(x, v, t)$ we have used so far. The Laplace transform of eq 3.2 is

$$
\begin{array}{r}
u P_{\alpha}\left(x, v, u, \gamma_{\alpha}\right)-P_{\alpha}\left(x, v, t=0, \gamma_{\alpha}\right)+v \frac{\partial P_{\alpha}\left(x, v, u, \gamma_{\alpha}\right)}{\partial x}+ \\
\frac{F(x)}{M} \frac{\partial P_{\alpha}\left(x, v, u, \gamma_{\alpha}\right)}{\partial v}=\gamma_{\alpha} u^{1-\alpha} \hat{L}_{\mathrm{fp}} P_{\alpha}\left(x, v, u, \gamma_{\alpha}\right)
\end{array}
$$

and $P_{\alpha}\left(x, v, u, \gamma_{\alpha}\right)$ is the Laplace transform of $P_{\alpha}\left(x, v, t, \gamma_{\alpha}\right)$. From eq 3.3 we learn that $P_{\alpha}\left(x, v, u, \gamma_{\alpha}\right)$ solves an ordinary Kramers equation in which the damping coefficient $\gamma_{1}$ was transformed according to

$$
\gamma_{1} \rightarrow \gamma_{\alpha} u^{1-\alpha}
$$

We therefore find

$$
P_{\alpha}\left(x, v, u, \gamma_{\alpha}\right)=P_{1}\left(x, v, u, \gamma_{\alpha} u^{1-\alpha}\right)
$$

assuming the initial conditions are identical for both solutions. Transforming to the time domain, we find the formal solution

$$
P_{\alpha}\left(x, v, t, \gamma_{\alpha}\right)=\mathrm{L}^{-1}\left[P_{1}\left(x, v, u, \gamma_{\alpha} u^{1-\alpha}\right)\right]
$$

with $\mathrm{L}^{-1}$ being the inverse Laplace transform. Closed-form solutions of an ordinary Kramers equation, $P_{1}\left(x, v, t, \gamma_{1}\right)$, are known only for a handful of cases; approximate solutions can be found using methods specified in ref 30 . In some cases eq 3.6 can be used to find moments of the solution of fractional Kramers equation, $\left\langle x^{n} v^{m}\right\rangle$, in a straightforward way. In what follows we shall revert to the notation $P(x, v, t)$ instead of $P_{\alpha}\left(x, v, t, \gamma_{\alpha}\right)$.

A. Force-Free Case 1. We consider the force-free case $F(x)$ $=0$. As usual $\langle\dot{x}(t)\rangle=\langle v(t)\rangle$, with the mean velocity $\langle v(t)\rangle$ given in eq 2.14; hence

$$
\langle x(t)\rangle=v_{0} t E_{\alpha, 2}\left(-\gamma_{\alpha} t^{\alpha}\right)
$$

where

$$
E_{\alpha, \beta}(z)=\sum_{k=0}^{\infty} \frac{z^{k}}{\Gamma(\alpha+\beta k)}
$$

is a generalized Mittag-Leffler function ${ }^{42}$ satisfying

$$
E_{\alpha, \beta}(z)=-\sum_{n=1}^{N-1} \frac{z^{-n}}{\Gamma(\beta-\alpha n)}+0\left(z^{-n}\right)
$$

with $z \rightarrow \infty$. For short times

$$
\langle x(t)\rangle \sim v_{0} t
$$

as expected for a pure ballistic propagation, and for long times

$$
\langle x(t)\rangle \sim \frac{v_{0} t^{1-\alpha}}{\gamma_{\alpha} \Gamma(2-\alpha)}
$$

The particle exhibits a net drift in a direction determined by the initial velocity $v_{0}$. Of course, when averaging over initial conditions, using thermal equilibrium condition, no net drift is observed as expected from symmetry. The mean-square dis- 
placement is determined by $\left\langle\dot{x}^{2}(t)\right\rangle=\langle 2 x(t) v(t)\rangle$. A short calculation using the Laplace transform of eq 3.2 shows

$$
\left\langle x^{2}(t)\right\rangle_{\mathrm{eq}}=2 \frac{k_{\mathrm{b}} T}{M} t^{2} E_{\alpha, 3}\left(-\gamma_{\alpha} t^{\alpha}\right)
$$

where the subscript eq means that thermal initial conditions are considered (i.e., $\left\langle v_{0}^{2}\right\rangle_{\mathrm{eq}}=k_{\mathrm{b}} T / M$ ). For short times

$$
\left\langle x^{2}(t)\right\rangle_{\mathrm{eq}} \sim \frac{k_{\mathrm{b}} T}{M} t^{2}
$$

while for long times

$$
\left\langle x^{2}(t)\right\rangle_{\mathrm{eq}} \sim 2 D_{\alpha} t^{2-\alpha}
$$

where

$$
D_{\alpha}=\frac{k_{\mathrm{b}} T}{\gamma_{\alpha} M \Gamma(3-\alpha)}
$$

Equation 3.14 exhibits an enhanced diffusion when $0<\alpha<1$. Equation 3.15 is the (first) generalized Einstein relation, and when $\alpha=1$, we recover the well-known Einstein relation $D_{1}$ $=k_{\mathrm{b}} T / M \gamma_{1}$.

It is straightforward to prove the more general Einstein relation

$$
\left\langle x^{2}(t)\right\rangle_{\mathrm{eq}}=2 \int_{0}^{t} \mathrm{~d} t^{\prime} \int_{0}^{t^{\prime}}\langle v(\tau) v(0)\rangle_{\mathrm{eq}} \mathrm{d} \tau
$$

where according to eq 2.14

$$
\langle v(\tau) v(0)\rangle_{\mathrm{eq}}=\frac{k_{\mathrm{b}} T}{M} E_{\alpha}\left(-\gamma \tau^{\alpha}\right)
$$

is the velocity autocorrelation function. We note that relation equation (3.16) is valid for stationary processes, ${ }^{43}$ while the collision model we investigated in the previous section is nonstationary.

Now consider the constant force field $F(x)=F$, using the fractional Kramers equation (3.2). We can show that a second generalized Einstein relation between the drift in the presence of the driving field, $\langle x(t)\rangle_{\mathrm{F}}$, and the mean-square displacement (eq 3.12), in the absence of the field is valid

$$
\langle x(t)\rangle_{\mathrm{F}}=F \frac{\left\langle x^{2}(t)\right\rangle_{\mathrm{eq}}}{2 k_{\mathrm{b}} T}
$$

This relation suggests that the fractional Kramers equation is compatible with linear response theory. ${ }^{44,45}$

B. Force-Free Case 2. We shall now use the formal solution equation (3.6) to find moments $\left\langle x^{2 n}(t)\right\rangle_{\mathrm{eq}}$ for the case $F(x)=0$. Odd moments are equal to zero. Consider the reduced probability density $W_{\text {eq }}(x, t)$ of finding the particle on $x$ at time $t$, defined according to

$$
W_{\text {eq }}(x, t)=\int_{-\infty}^{\infty} \mathrm{d} v \int_{-\infty}^{\infty} \mathrm{d} v_{0} P(x, v, t) M\left(v_{0}\right)
$$

$P(x, v, t)$ is the solution of the fractional Kramers equation with initial conditions concentrated on $x_{0}$ and $v_{0} . M\left(v_{0}\right)$ is Maxwell's probability density, implying an equilibrium initial condition for the initial velocity $v_{0}$.
For the standard case $\alpha=1, W_{\text {eq }}(x, t)$ is a Gaussian; therefore

$$
\left\langle x^{2 n}(t)\right\rangle_{\mathrm{eq}}=\int_{-\infty}^{\infty} x^{2 n} W_{\mathrm{eq}}(x, t) \mathrm{d} x=\frac{(2 n) !}{2^{n} n !}\left\langle x^{2}(t)\right\rangle_{\mathrm{eq}}^{n}
$$

and according to ref 30

$$
\left\langle x^{2}(t)\right\rangle_{\mathrm{eq}}=2 \frac{k_{\mathrm{b}} T\left[\gamma_{1} t-1+\exp \left(-\gamma_{1} t\right)\right]}{M} \gamma_{1}^{2}
$$

which in the long-time limit gives (only for $\alpha=1$ )

$$
\left\langle x^{2}(t)\right\rangle_{\mathrm{eq}} \sim 2 \frac{k_{\mathrm{b}} T}{M \gamma_{1}} t
$$

According to eqs $3.4-3.6$ the calculation of $\left\langle x^{2 n}(t)\right\rangle_{\mathrm{eq}}$, for 0 $<\alpha<1$, follows three steps. First, find the Laplace transform of the Gaussian moments, $\left\langle x^{2 n}(t)\right\rangle_{\mathrm{eq}}$ (eq 3.20). Because we shall be interested in the long time behavior of $\left\langle x^{2 n}(t)\right\rangle_{\mathrm{eq}}$, it is sufficient to consider only the long-time behavior of the standard $\alpha=1$ case; namely, we use the asymptotic eq 3.22 instead of eq 3.21. We note that the inclusion of the short-time behavior is also straightforward but of less interest to us here. It is easy to show that

$$
\left\langle x^{2 n}(u)\right\rangle_{\mathrm{eq}} \sim(2 n) !\left(\frac{k_{\mathrm{b}} T}{M \gamma_{1}}\right)^{n} \frac{1}{u^{n+1}}
$$

is valid for small $u$ and $\alpha=1$. The second step is to transform eq 3.23 using eq 3.4 and the last step is to invert Laplace transform, the result from the second step, as in eq 3.6. We find

$$
\left\langle x^{2 n}(t)\right\rangle_{\mathrm{eq}} \sim(2 n) !\left(\frac{k_{\mathrm{b}} T}{M \gamma_{\alpha}}\right)^{n} \frac{t^{n(2-\alpha)}}{\Gamma(2 n-n \alpha+1)}
$$

It is easy to check that the moments in eq 3.24 can also be calculated based on

$$
\left\langle x^{2 n}(t)\right\rangle \sim \mathrm{L}^{-1}\left[\left(\frac{d}{i d k}\right)^{2 n}\left(\frac{u^{1-\alpha}}{u^{2-\alpha}+\frac{k_{\mathrm{b}} T}{M \gamma_{\alpha}} k^{2}}\right) \mid k=0\right]
$$

This result is also to be expected. In Fourier-Laplace space the well-known $\alpha=1$ solution is

$$
W_{\mathrm{eq}}(k, u) \sim \frac{1}{u+\frac{k_{\mathrm{b}} T}{M \gamma_{1}} k^{2}}
$$

If we use the transformation (3.4) in eq 3.26, we find

$$
W_{\mathrm{eq}}(k, u) \sim \frac{u^{1-\alpha}}{u^{2-\alpha}+\frac{k_{\mathrm{b}} T}{M \gamma_{\alpha}} k^{2}}
$$

This is the moment generating function in eq 3.25. We note that, in general, there is no guarantee that the transformation eq 3.4 can be made after the small $u$ limit of the $\alpha=1$ solution is taken; instead the small $u$ limit must be taken only after the transformation eq 3.4 takes place. The small $(k, u)$ expansion, of a particular coupled CTRW also known as Lévy walks, has the same form as eq 3.27 see eq 38 in ref 46 \}. 
According to eq $3.27, W_{\mathrm{eq}}(x, t)$ satisfies the fractional diffusion equation

$$
\frac{\partial W_{\mathrm{eq}}(x, t)}{\partial t}=\frac{k_{\mathrm{b}} T}{M \gamma_{\alpha}}{ }_{0} D_{t}^{-(1-\alpha)} \frac{\partial^{2}}{\partial x^{2}} W_{\mathrm{eq}}(x, t)
$$

which is expected to work well only at large times. Equation 3.28 was investigated by Schneider and Wyss. ${ }^{8}$

For one dimension the inverse Laplace-Fourier transform of eq 3.27 is

$$
W_{e q}(x, t) \sim \frac{\sqrt{\gamma_{\alpha} M}}{\sqrt{2 k_{\mathrm{b}} T}(1-\alpha / 2)}\left(\frac{z^{2-\alpha / 2}}{t^{1-\alpha / 2}}\right) l_{1-\alpha / 2}(z)
$$

with

$$
z=t\left[\frac{\sqrt{k_{\mathrm{b}} T}}{\left(\sqrt{2 M \gamma_{\alpha}}|x|\right)}\right]^{1 /(1-\alpha / 2)}
$$

The inversion of the two- and three-dimensional solutions is not as straightforward as that for the one-dimensional case, and we leave the details for a future publication.

C. Collision Model. We have derived the fractional FokkerPlanck equation (2.10) from a nonstationary stochastic collision model in the limit of $\epsilon=m / M \rightarrow 0$. Equation 2.10 describing a fractional Ornstein-Uhlenbeck process is the basis of the fractional Kramers equation, which is reached after adding the standard streaming terms (i.e., Newton's law of motion). In refs 27 and 28 the mean-square displacement, $\left\langle x^{2}(t)\right\rangle_{\mathrm{col}}$, of the test particle was calculated also for mass ratios $\epsilon$ not tending to zero. It was shown that the collision process gives $\left\langle x^{2}\right\rangle_{\text {col }} \sim t^{2}$ for 0 $<\alpha<1$ and finite $\epsilon$, a behavior different from the asymptotic behavior we have found in eq 3.14 based on the fractional Kramers equation, $\left\langle x^{2}\right\rangle \sim t^{2-\alpha}$. The $\left\langle x^{2}\right\rangle_{\mathrm{col}} \sim t^{2}$ behavior of the collision model can be understood by the fact that within the collision process, one typically finds long (collision-free) time intervals, on the order of the observation time $t$, in which the motion is ballistic so that $x^{2} \sim t^{2}$. A similar ballistic behavior is known from the Lévy walk model with diverging time intervals between turning points. As $\epsilon$ becomes smaller, the correction to the ballistic term becomes important for longer times and these correction terms behave like $\left\langle x^{2}\right\rangle \sim t^{2-\alpha}$. Also it is clear that the two limits of $t \rightarrow \infty$ and $\epsilon \rightarrow 0$ do not commute. If we first take $t \rightarrow \infty$ and only then $\epsilon \rightarrow 0$, we find

$$
\left\langle x^{2}(t)\right\rangle_{\mathrm{col}} \sim t^{2}
$$

Hence, the derivation of the fractional Kramers equation from the stochastic collision model is a delicate matter, with a result depending on the order in which limits are taken. Another difficulty in our derivation is that our starting point is a nonstationary process. It is still unclear if stationary models can lead to dynamics described by the fractional Kramers equation.

\section{Summary and Discussion}

We have investigated a fractional Kramers equation which has the following properties: (a) the velocity of the particle evolves according to a fractional Ornstein-Uhlenbeck process described by eq 2.10 ; the velocity moments decay according to a Mittag-Leffler relaxation, namely, as a stretched exponential (Kohlrausch form) for short times and as a power law for long times; (b) in the absence of a force field diffusion is enhanced, $1<\mathrm{d}<2$; (c) the stationary solution of the fractional Kramers equation is the Maxwell-Boltzmann distribution; (d) Einstein relations are obeyed in consistency with fluctuation dissipation theorem and linear response theory, (e) in Laplace space a simple transformation of solutions of an ordinary Kramers equation gives the solution of a fractional Kramers equation.

As mentioned in the Introduction, fractional kinetic equations in the literature are related to the CTRW. We showed here that in the small $(k, u)$ limit $W_{\text {eq }}(k, u)$ has the same form as a particular coupled Levy walk CTRW. Other limits of the CTRW are shown to correspond to other fractional kinetic equations. As pointed out in ref 22 , the fractional diffusion equation, ${ }^{8}$ describing the subdiffusion, corresponds to the uncoupled CTRW in the limit of small $(k, u)$. A comparison between the uncoupled CTRW and solution of the fractional diffusion equation in $(x, t)$ space was carried out in ref 24. Another fractional equation in refs 10 and 11 describes Lévy flights for which $\left\langle x^{2}\right\rangle=\infty$; such a fractional equation is related to the decoupled limit of the CTRW with diverging jump lengths.

One may ask if it is worthwhile to introduce fractional derivatives, given that the older CTRW approach is so successful. Besides the fact that fractional equations are beautiful and simple (i.e., in some cases they are solvable), these equations can incorporate the effect of an external potential field. To us, this extension seems important although not explored in depth in the present paper. Little is known on anomalous diffusion in an external force field.

Metzler et al. ${ }^{13}$ have investigated a fractional Fokker-Planck equation defined with the fractional Liouville-Riemann operator. In the absence of an external force, the fractional FokkerPlanck equation investigated in ref 13 describes a subdiffusive behavior $(\delta<1)$. The equation considers a type of overdamped dynamics in which only the coordinate $x$ is considered not the velocity $v$. The fractional Fokker-Planck equation in ref 13 together with the fractional Kramers equation investigated here gives a stochastic framework for both subdiffusion and enhanced diffusion in an external field. We believe that both approaches will find their application.

Note Added in Proof. Recently related work on fractional diffusion was published in ref 47.

Acknowledgment. E.B. thanks J. Klafter and R. Metzler for helpful discussions. This research was supported in part by a grant from the NSF.

\section{Appendix A}

We find the Laplace transform of eq 2.33

$$
R_{s}(u)=\int_{0}^{\infty} \mathrm{e}^{-u t} \frac{1}{\alpha \gamma_{\alpha} t^{\alpha}} z^{\alpha+1} l_{\alpha}(z) \mathrm{d} t
$$

with $z$ defined in eq 2.34. Using the change of variables $t=$ $y\left(s / \gamma_{\alpha}\right)^{1 / \alpha}$, it is easy to show $(s \neq 0)$

$$
\begin{aligned}
R_{s}(u)=- & \frac{1}{\alpha s} \frac{\mathrm{d}}{\mathrm{d} u} \int_{0}^{\infty} \exp \left[-y\left(\frac{s}{\gamma_{\alpha}}\right)^{1 / \alpha} u\right] l_{\alpha}(y) \mathrm{d} y= \\
& -\left(\frac{1}{\alpha s}\right) \frac{\mathrm{d}}{\mathrm{d} u} \exp \left(-\frac{s}{\gamma_{\alpha}} u^{\alpha}\right)=\frac{u^{\alpha-1}}{\gamma_{\alpha}} \exp \left(-\frac{s u^{\alpha}}{\gamma_{\alpha}}\right)(\text { A.2 })
\end{aligned}
$$

which is $R_{s}(u)$ (eq 2.31). From eq 2.29 we learn that eq 2.31 is valid also for $s=0$. 


\section{References and Notes}

(1) Scher, H.; Montroll, E. Phys. Rev. B 1975, 12, 2455.

(2) Scher, H.; Lax, M. Phys. Rev. B 1973, 7, 4491; Phys. Rev. B 1973, 7,4502 .

(3) Weiss, G. H. Aspects and Applications of the Random Walk; North Holland: Amsterdam, The Netherlands, 1994.

(4) Bouchaud, J.-P.; Georges, A. Phys. Rep. 1990, 195, 12. 33.

(5) Klafter, J.; Shlesinger, M. F.; Zumofen, G. Phys. Today 1996, 49,

(6) Balescu, R. Statistical Dynamics; Matter Out of Equilibrium; Imperial College Press and World Scientific: Singapore, 1997.

(7) Barkai, E.; Klafter, J. Chaos, Kinetics and Non-linear Dynamics in Fluids and Plasmas; Benkadda, S., Zaslavsky, G. M., Eds.; Lecture Notes in Physics; Springer-Verlag: Berlin, 1998.

(8) Schneider, W. R.; Wyss, W. J. Math. Phys. 1989, 30, 134 6426.

(9) Glöckle, W. G.; Nonnenmacher, T. F. Macromolecules 1991, 24

(10) Fogedby, H. C. Phys. Rev. Lett. 1994, 73, 2517; Phys. Rev. E 1998, 58, 1690. Jespersen, S.; Metzler, R.; Fogedby, H. C. Phys. Rev. E 1999, $59,2736$.

(11) Zaslavsky, G. M.; Edelman, M.; Niyazov, B. A. Chaos 1997, 7 , 159.

(12) Saichev, A. I.; Zaslavsky, M. Chaos 1997, 7, 4.

(13) Metzler, R.; Barkai, E.; Klafter, J. Phys. Rev. Lett. 1999, 82, 3563.

(14) Huillet, T. J. Phys. A 1999, 32, 7225.

(15) Grigolini, P.; Rocco, A.; West, B. J. Phys. Rev. E. 1999, 59, 2603.

(16) Kusnezov, D.; Bulgac, A.; Do Dang, G. Phys. Rev. Lett. 1999, 82, 1136.

(17) Zumofen, G.; Klafter, J. Chem. Phys. Lett. 1994, 219, 303.

(18) Barkai, E.; Silbey, R. Chem. Phys. Lett. 1999, 310, 287.

(19) Chernyak, V.; Schultz, M.; Mukamel, S. J. Chem. Phys. 1999, 111, 7416.

(20) Oldham, K. B.; Spanier, J. The Fractional Calculus; Academic Press: New York, 1974.

(21) Hilfer, R.; Anton, L. Phys. Rev. E. 1995, 51, R848.

(22) Compte, A. Phys. Rev. E 1996, 53, 4191.

(23) West, B. J.; Grigolini, P.; Metzler, R.; Nonnenmacher, T. F. Phys. Rev. E 1997, 55, 99.

(24) Barkai, E.; Metzler, R.; Klafter, J. Phys. Rev. E 2000, 61, 132.

(25) Lord Rayleigh Phil. Mag. 1891, 32, 424, [Sci. Pap. (Cambridge) 1902, 3, 473.]

(26) van Kampen, N. G. Stochastic Processes in Physics and Chemistry;

North Holland: Amsterdam, The Netherlands, 1981).

(27) Barkai, E.; Fleurov, V. N. J. Chem. Phys. 1996, 212, 69

(28) Barkai, E.; Fleurov, V. N. Phys. Rev. E. 1997, 56, 6355.
(29) Metzler, R.; Klafter, J., preprint

(30) Risken, H. The Fokker-Planck Equation; Springer: Berlin, 1989.

(31) To see this Laplace transform equation 2.10 using eq 2.7

$u Q(u, v)-Q(v, t=0)=\gamma_{\alpha} \hat{L}_{\mathrm{fp}}\left[u^{1-\alpha} Q(v, u)-\left.{ }_{0} D_{t}^{-\alpha} Q(v, t)\right|_{t=0}\right]$

setting $\left.{ }_{0} D_{t}^{-\alpha} Q(v, t)\right|_{t=0}=0$, we get an expression equivalent to the Laplace transform of eq 2.9 .

(32) Feller,W. An Introduction to Probability Theory and Its Applications; John Wiley and Sons: New York, 1970; Vol. 2.

(33) Zoltarev, V. M. Dokl. Acad. Nauk. USSR 1954, 98, 715.

(34) Montroll, E. W.; West, B. J. In Fluctuation Phenomena; Montroll, E. W., Lebowitz, J. L., Eds.; North-Holland: Amsterdam, The Netherlands, 1987. 251.

(35) Hänggi, P.; Talkner, P.; Borkovec, M. Rev. Mod. Phys. 1990, 62,

(36) Montgomery, J. A.; Chandler, D.; Berne, B. J. J. Chem. Phys. 1979, $70,4056$.

(37) Knessel, C.; Mangel, M.; Matkowsky, B. J.; Schuss, A.; Tier, C. J. Chem. Phys. 1984, 81, 1285.

(38) Borkovek, M.; Straub, J. E.; Berne, B. J. J. Chem. Phys. 1986, 85, 146.

(39) Barkai, E.; Fleurov, V. Phys. Rev. E 1995, 52, 1558.

(40) Bicout, D. J.; Berezhkovskii, A. M.; Szabo, A.; Weiss, G. H. Phys. Rev. E 1999, 59, 3702 .

(41) Berezhkovskii, A. M.; Bicout, D. J.; Weiss, G. H. J. Chem. Phys 1999, 111, 11050

(42) Erdelyi, A., Ed. Higher Transcendental Functions; McGraw-Hill: New York, 1955.

(43) To see this, write

$$
\left\langle x^{2}(t)\right\rangle_{\mathrm{eq}}=\left\langle\left[\int_{0}^{t} v\left(t_{1}\right) \mathrm{d} t_{1}\right]\left[\int_{0}^{t} v\left(t_{2}\right) \mathrm{d} t_{2}\right]\right\rangle_{\mathrm{eq}}
$$

if the process is stationary $\left\langle v\left(t_{1}\right) v\left(t_{2}\right)\right\rangle_{\mathrm{eq}}=\langle v(\tau) v(0)\rangle_{\mathrm{eq}}$ with $\tau=\left|t_{1}-t_{2}\right|$, and only then eq 3.16 is valid.

(44) Barkai, E.; Fleurov, V. N. Phys. Rev. E 1998, 58, 1296.

(45) Berlin, Y. A.; Siebbeles, L. D. A.; Zharikov, A. A. Chem. Phys. Lett. 1999, 305, 123.

(46) Klafter, J.; Blumen, A.; Shlesinger, M. F. Phys. Rev. A 1987, 35, 3081 .

(47) Bologna, M.; Grigolini, P.; Riccardi, J. Phys. Rev. E 1999, 60, 6453. Kutner, R.; Wysocki, K. Physica A 1999, 274, 67. Yanovsky, V. V.; Chechkin, A. V.; Schertzer, D.; Tour, A. V. nlin/001035 (to appear in Physica A). 